\title{
El Boletín de la Sociedad Botánica de México en el contexto de las publicaciones científicas
}

\author{
ANTONIO LOT Y ARMANDO BUTANDA \\ Departamento de Botánica, Instituto de Biología, UNAM, Apdo. Postal 70-233, Coyoacán. 04510, \\ México, D.F.
}

\begin{abstract}
Resumen. Se reseña cuál ha sido el papel del Boletín de la Sociedad Botánica de México, como una de las publicaciones científicas de mayor tradición en el desarrollo de la ciencias vegetales de México. A lo largo de sus primeros 50 años en el Boletín se han publicado 396 artículos en 54 números. Al revisar el contenido de éstos, se aprecia la preferencia de los autores y la selección de los editores por temas taxonómicos, seguidos por los florísticos, ecológicos, fitogeográficos y etnobotánicos. Un análisis de la última década (a partir del número 44) revela que en los numerosos artículos taxonómicos se han descrito un género y 34 especies de plantas nuevos para la ciencia, lo que ha contribuido al conocimiento de la diversidad de la flora de México.
\end{abstract}

Abstract. A review of the role played by the Boletín de la Sociedad Botánica de México reveals it to be one of the leading scientific publications of long standing tradition in the development of plant sciences in Mexico. In its 50 years of existence, the Boletín has published 396 papers in 54 numbers. When these are evaluated, the preference of authors and the inclination of editors in selecting papers are principally directed toward taxonomic topics, followed by floristic studies, ecology, phytogeography and ethnobotany. An analysis of the last decade (beginning with number 44) shows that one genus and 34 species of plants new to science have described in the numerous taxonomic papers, thus contributing to the knowledge of the diversity of the flora of Mexico.

\section{INTRODUCCIÓN}

Diversos autores activos en investigación científica, y personas experimentadas en el oficio editorial de revistas científicas, han publicado y presentado en diferentes foros sus puntos de vista sobre la problemática y el significado del sostenimiento y difusión de las llamadas publicaciones periódicas primarias, muy especialmente aquellas que dependen de sociedades científicas.

Como ejemplo, mencionaremos dos reuniones: la primera, acerca de los Sistemas de Información Científica y Técnica de México, con motivo de una Mesa Redonda en el Coloquio sobre Políticas Nacionales en Ciencia y Tecnología, cuyos resultados fueron publicados en 1972 por la Academia de la Investigación Científica, A.C. (Bolívar et al., 1972); la segunda, el Seminario sobre Revistas Científicas Nacionales, realizado en 1972, cuyas Memorias fueron publicadas al año siguiente por el Consejo Nacional de Ciencia y Tecnología (Benítez Bribiesca et al., 1973).

Con relación a lo anterior, es interesante mencionar que la mayoría de las inquietudes y perspectivas sobre la política editorial y el desarrollo de revistas científicas mexicanas de calidad en un plano internacional, siguen vigentes después de más de dos décadas.

A fines de 1986, el Colegio de México también organizó un ciclo de conferencias: Uso de la Información Científica en México, publicadas dos años después por el Centro de Investigaciones Interdisciplinarias en Humanidades, Universidad Nacional Autónoma de México (UNAM) (Pérez Tamayo, 1988).
En la colección Biblioteca del Editor, la Coordinación de Humanidades de la UNAM publicó entre otros, el volumen Arte Editorial en la Literatura Científica (Sánchez y Gándara et al., 1992). En uno de sus capítulos se trata lo relativo a las revistas científicas mexicanas y de cómo «...nacen fecundadas por necesidades gremiales, institucionales o personales... mueren generalmente por inanición, cuyos síntomas visibles son falta de autores y de lectores (y de citas); no necesariamente por falta de excelencia». En otra parte, los autores mencionan que «las revistas vigorosas no son producto artesanal de un grupo de investigadores entusiastas; son hechas profesionalmente».

Larqué Saavedra $(1993,1994)$ en sus artículos sobre las sociedades científicas en México y sobre las revistas de ciencias naturales, resalta el papel que han desempeñado en el desarrollo de las ciencias agrícolas.

Todos estos esfuerzos de análisis y de discusión entre expertos, reflejan un legítimo interés por avanzar en la comprensión del problema y en la búsqueda de diversas salidas para superarlo en cada área del conocimiento.

Tomando como marco de referencia las perspectivas de las ciencias vegetales, es fundamental preguntarnos cuál ha sido la participación de las publicaciones científicas en la maduración y los logros del desarrollo de la botánica en México.

Lo anterior nos lleva a intentar ubicar objetivamente al Boletín de la Sociedad Botánica de México dentro del contexto de las revistas científicas, con las que comparte limitaciones, y que presenta además algunos de los problemas propios de las sociedades científicas en cuanto a su 
financiamiento e infraestructura editorial. Sin embargo, es justo destacar que salvo unos cuantos años aislados, el Boletín ha sido publicado regularmente desde hace medio siglo (Cuadro 1).

CuAdro 1. Cronología en su publicación

\begin{tabular}{|c|c|c|c|}
\hline Boletín No. & Fechadeseriación & Boletín No. & Fechadeseriación \\
\hline 1 & enero, 1944 & 28 & septiembre, 1963 \\
\hline 2 & enero, 1945 & 29 & julio, 1965 \\
\hline 3 & octubre, 1945 & 30 & mayo, 1969 \\
\hline 4 & mayo, 1946 & 31 & diciembre, 1970 \\
\hline 5 & mayo, 1947 & 32 & diciembre, 1972 \\
\hline 6 & marzo, 1948 & 33 & enero, 1974 \\
\hline 7 & octubre, 1948 & 34 & marzo, 1975 \\
\hline 8 & abril, 1949 & 35 & diciembre, 1975 \\
\hline 9 & octubre, 1949 & 36 & diciembre, 1977 \\
\hline 10 & abril, 1950 & 37 & diciembre, 1977 \\
\hline 11 & diciembre, 1950 & 38 & diciembre, 1979 \\
\hline 12 & junio, 1951 & 39 & junio, 1980 \\
\hline 13 & diciembre, 1951 & 40 & octubre, 1981 \\
\hline 14 & junio, 1952 & 41 & noviembre, 1981 \\
\hline 15 & junio, 1953 & 42 & agosto, 1982 \\
\hline 16 & febrero, 1954 & 43 & diciembre, 1982 \\
\hline 17 & noviembre, 1954 & 44 & diciembre, 1983 \\
\hline 18 & julio, 1955 & 45 & diciembre, $1983^{1}$ \\
\hline 19 & julio, 1956 & 46 & junio, $1984^{2}$ \\
\hline 20 & marzo, 1957 & 47 & julio, 1987 \\
\hline 21 & agosto, 1957 & 48 & diciembre, $1983^{3}$ \\
\hline 22 & enero, 1958 & 49 & diciembre, 1989 \\
\hline 23 & diciembre, 1958 & 50 & diciembre, 1990 \\
\hline 24 & noviembre, 1959 & 51 & diciembre, 1991 \\
\hline 25 & agosto, 1960 & 52 & diciembre, 1992 \\
\hline 26 & julio, 1961 & 53 & diciembre, 1993 \\
\hline 27 & noviembre, 1962 & 54 & junio, 1994 \\
\hline
\end{tabular}

${ }^{1}$ Fecha de publicación, julio, 1984; ${ }^{2}$ junio, $1986 ;{ }^{3}$ 10, mayo, 1989.
En el presente artículo trataremos de bosquejar algunas reflexiones sobre el Boletín de la Sociedad Botánica de México en el contexto de las publicaciones científicas, con motivo del quincuagésimo aniversario de su aparición, que celebramos con la edición de este número especial.

\section{ANTECEDENTES, CARACTERÍSTICAS Y ESTRUCTURA}

En 1944, a tres años de la fundación de la Sociedad Botánica de México, y siendo presidente en la mesa directiva Maximino Martínez, se edita el primer número del Boletín (fig. 1) que contiene dos artículos breves, notas bibliográficas, actividades de los socios y la lista de los miembros de la Sociedad, que en ese tiempo era de cinco honorarios, 26 titulares y 17 corresponsales (Lot y Carvajal, 1981).

A partir del número 21 de 1957, y hasta el 28 de 1963, el Boletín es apoyado económicamente por el Instituto Nacional de Investigación Científica (antecedente del Consejo Nacional de Ciencia y Tecnología CONACyT). Desde el número 34 de 1975 hasta la fecha, se imprime con la ayuda de un subsidio (en 1983, Convenio) del CONACyT.

En 1960, durante la presidencia de Jerzy Rzedowski en la Mesa Directiva, se nombra oficialmente el primer comité editorial, integrado por Maximino Martínez, Efraím Hernández Xolocotzi, Gastón Guzmán y Faustino Miranda.

Desde 1968, el Boletín se complementa con la edición de un órgano informativo de la Sociedad, el cual desde 1970 lleva el nombre de Macpalxóchitl, a partir del número 23 (Butanda, 1989). De esta manera, la publicación de noticias, artículos de divulgación y diversos ensayos, que regularmente no tienen cabida en el boletín científico, ha permitido ampliar su efecto de comunicación hacia estudiantes y aficionados a la botánica.
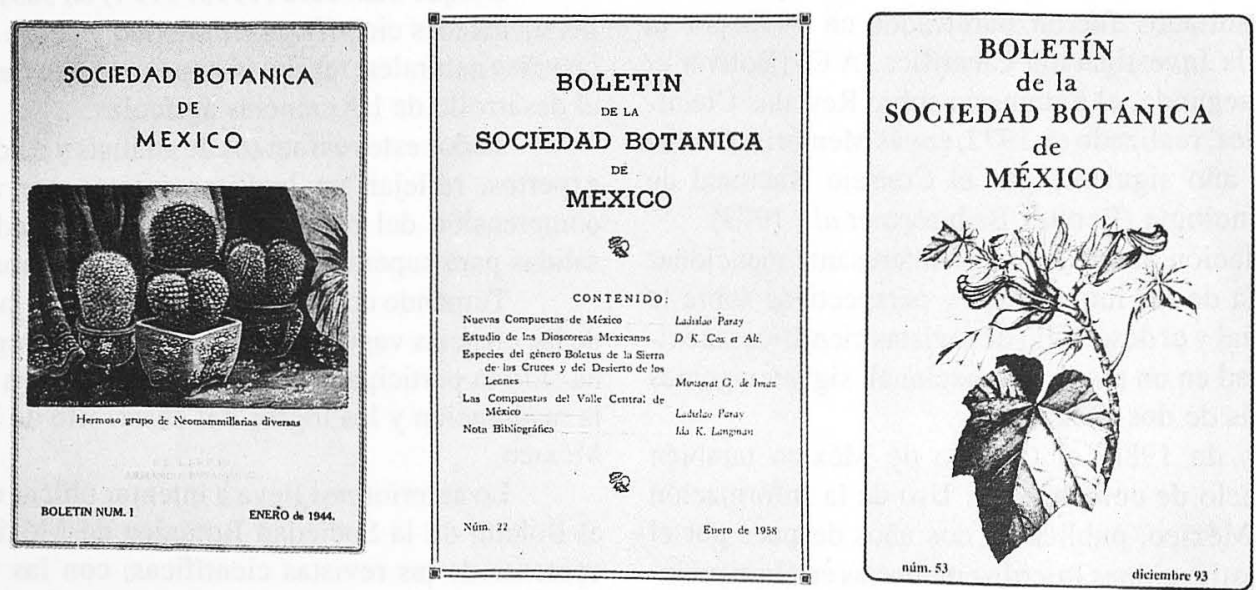

FIG. 1. Primer número y cambios ocurridos en su portada. 
El Boletín está abierto a trabajos que sean el resultado de investigaciones científicas originales e inéditas, que se refieran a aspectos de la botánica en México, estudios comparativos de otras regiones con México, o estudios teóricos y metodológicos de alto interés universal para los botánicos en general.

Además de los números anuales regulares, se editan algunos especiales en ocasión de diversas reuniones que tienen un alto significado en el desarrollo científico, el avance de la botánica nacional y las perspectivas de las ciencias vegetales, como es el caso de este número.

Los manuscritos se aceptan en función de los criterios de originalidad, rigor científico y contribución en su campo. La evaluación de los manuscritos sometidos se realiza en apego a las normas editoriales establecidas por el Comité Editorial con el auxilio de los revisores externos (árbitros). Entre los miembros que por varias décadas, en diferentes combinaciones, han formado parte del Comité Editorial, se encuentran Maximino Martínez, Faustino Miranda,EfraímHernándezXolocotzin,Jerzy Rzedowski,Arturo Gómez Pompa, E. Mark Engleman, José Sarukhán y Carlos Vázquez-Yanes (Cuadro 2).

Desde hace tres lustros, el número de artículos promedio que se publica al año es entre 10 y 15 , representando de 100 a 150 páginas impresas. El porcentaje promedio de rechazo es de $10 \%$.

Un aspecto interesante en su contenido ha sido la publicación como artículos científicos de los temas de numerosas tesis profesionales y de grado.

La distribución del Boletín a 42 bibliotecas de instituciones nacionales y a 97 centros de investigación en el extranjero, junto con su inclusión en 12 índices internacionales (publicaciones secundarias), ha permitido que la información contenida llegue a un número apreciable de lectores especialmente en los Estados Unidos de América y en Latinoamérica.

\section{IMPACTO DEL BOLETÍN}

No obstante la incertidumbre propia de una revista que depende de una sociedad científica, el Boletín no ha dejado de salir prácticamente desde 1944, cumpliendo con la publicación y difusión de, quizá, las más importantes contribuciones en cada etapa de la vida de la comunidad botánica de México. Otras dos revistas con las que se complementa la publicación del quehacer botánico en México son Anales del Instituto de Biología de la Universidad Nacional Autónoma de México, Serie Botánica y Acta Botánica Mexicana del Instituto de Ecología. Ambas dependen de instituciones de investigación que usualmente cuentan con mayores recursos y cierta infraestructura; sin embargo, el Boletín de la Sociedad Botánica de México puede ser justa y objetivamente considerado como una revista de primera al igual que las otras dos mencionadas. En ellas se publican prácticamente todos los resultados de las investigaciones originales de los botánicos que estudian la diversidad vegetal del te-
Cuadro 2. Lista de los miembros del Comité Editorial en diferentes épocas ${ }^{1}$.

\begin{tabular}{|c|c|c|}
\hline Período No. & Años & Miembros del Comité Editorial \\
\hline $1960-1964$ & (5) & Maximino Martínez ${ }^{2}$ \\
\hline $1960-1964$ & (5) & Faustino Miranda \\
\hline $1960-1991$ & $(30)$ & Efraím Hernández X. \\
\hline $1960-1961$ & (4) & Gastón Guzmán H. \\
\hline \multicolumn{3}{|l|}{$1976-1977$} \\
\hline $1962-1963$ & (2) & Jesús Vázquez Soto \\
\hline $1962-1969$ & (8) & Ramón Riba \\
\hline $1968-1984$ & (17) & Jerzy Rzedowski \\
\hline $1968-1973$ & $(5)$ & Arturo Gómez Pompa \\
\hline $1970-1973$ & (4) & Roberto Cruz Cisneros \\
\hline 1974-1979 & (6) & Mario Sousa Sánchez \\
\hline 1974-1975 & $(2)$ & Claudio Delgadillo $\mathrm{M}$. \\
\hline 1976-1993 & (17) & E. Mark Engleman \\
\hline 1976-1984 & (9) & José Sarukhán K. \\
\hline 1976-1977 & (12) & Armando Butanda C. \\
\hline \multicolumn{3}{|l|}{$1985-1994$} \\
\hline $1978-1979$ & (2) & Aurora Chimal H. \\
\hline $1978-1984$ & $(7)$ & Víctor Toledo M. \\
\hline $1980-1984$ & (5) & Carlos Vázquez Yanes \\
\hline $1980-1981$ & (2) & Victoria Sosa \\
\hline 1982-1994 & $(13)$ & Rosaura Grether G. \\
\hline $1982-1984$ & (3) & L. Ferreyra \\
\hline 1985-1994 & $(10)$ & Antonio Lot Helgueras \\
\hline $1985-1994$ & $(10)$ & Rodolfo Dirzo Minjares \\
\hline $1985-1994$ & $(10)$ & Hermilo Quero Rico \\
\hline $1985-1987$ & (3) & Armando Rodríguez Issasi \\
\hline $1988-1990$ & (3) & Ana Mendoza Ochoa \\
\hline 1991-1994 & (4) & Arturo Argueta \\
\hline 1991-1993 & (3) & Graciela Zamudio Varela \\
\hline 1994- & (1) & Ken Oyama \\
\hline
\end{tabular}

${ }^{1} \mathrm{La}$ información fue tomada de la segunda de forros del Boletín.

${ }^{2}$ No sólo fundó la Sociedad, él fue quién se encargó de la edición hasta su muerte.

rritorio nacional, en equilibrio con unaamplialista de revistas extranjeras especializadas.

Un ejemplo que ilustra bien el impacto de algunas contribuciones es el artículo de Miranda y Hernández X., publicado en el número 28 de 1963, «Tipos de vegetación de México y su clasificación», que ha sido citado más de un millar de veces por autores nacionales y extranjeros que estudian aspectos de ecología, florística y biogeografía, especialmente antes de la aparición del libro Vegetación de México de Rzedowski.

Este artículo, clásico en la biología mexicana, al igual que el de Rzedowski publicado en el número 29 de 1965, «Relaciones geográficas y posibles orígenes de la flora de México», fueron reimpresos varias veces por la demanda de colegas y estudiosos.

Si bien no está certificado el número de veces que éstos y otros artículos del Boletín han sido considerados, y aun citados en trabajos publicados en revistas periódicas, es evidente su significado en el área del conocimiento de una amplia gama de disciplinas botánicas. 
Asimismo, a través de sus índices acumulativos (de los números 1-50), se observa que el conocimiento de la flora y vegetación de México ha sido una de las áreas más consistentes, donde numerosos investigadores han contribuido con sus preguntas, hipótesis, ensayos, análisis y resultados.

También es justo mencionar que el Boletín fue evaluado positivamente por el Consejo Nacional de Ciencia y Tecnología, en la convocatoria que abrió a la comunidad científica en abril de 1993 sobre Evaluación de Revistas Científicas Mexicanas. En otras palabras, su registro en el Índice de Revistas Científicas Mexicanas de Excelencia, al lado de otras en el área de botánica, que resultaron con atributos de calidad, señalan al Boletín como una revista que ha hecho un esfuerzo sostenido por mejorar su contenido y elevar en general la calidad de la publicación científica.

\section{CONTRIBUCIÓN DEL BOLETÍN EN LAS CIENCIAS VEGETALES}

Las áreas que tradicionalmente agrupa el Boletín, y que lo conforman regularmente, son taxonomía y florística, fitogeografía, ecología (biología floral y reproductiva, análisis de vegetación, ecología de poblaciones, ecofisiología), etnobotánica, anatomía y morfología, ficología, micología, fitoquímica, genética, e historia de la botánica, entre otras. La figura 2 muestra el análisis de los temas cubiertos por décadas.

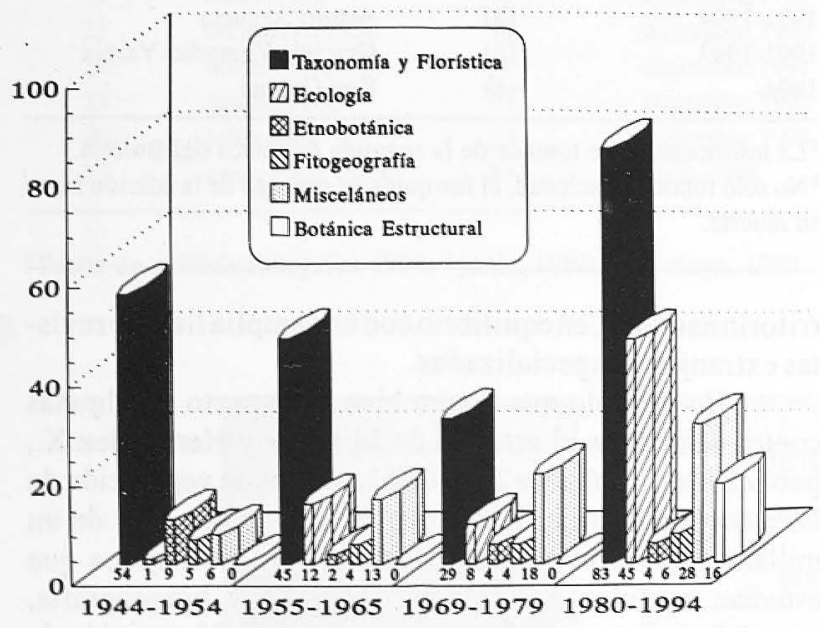

FIg. 2. Temas cubiertos durante cuatro décadas. Botánica estructural incluye los trabajos sobre anatomía, morfología y morfogénesis

Autores nacionales y extranjeros han publicado en el Boletín un centenar de nuevos taxa y numerosas revisiones taxonómicas de plantas mexicanas

Una lectura que puede resultar de utilidad al lector de este artículo, interesado en conocer con cierto detalle el análisis que parte de 26 títulos de revistas periódicas en el área de botánica, en un sentido amplio, se encuentra en el número 133 del Macpalxóchitl (Lot, 1994). En este artículo, además de mencionar los parámetros de evaluación de las revistas científicas, se presentan datos sobre las características de las principales publicaciones y su aporte en artículos y temas botánicos a lo largo de su existencia. Esto se detalla particularmente para las revistas Acta Botánica, Anales del Instituto de Biología, Serie Botánica y Boletín de la Sociedad Botánica de México.

El análisis que se hizo para efecto del presente artículo, está representado en los cuadros 3 y 4 , en los que se encuentran algunos elementos que pueden ser importantes en la función e impacto del Boletín, como medio de publicación de los resultados de científicos y aficionados de la botánica mexicana.

Cuadro 3. Número de artículos publicados y volúmenes editados por las tres revistas con mayor regularidad en su aparición

Período de las revistas Artículos publicados Vol.editados(años)

Bol.Soc.Bot.Méx.

396

$54(50)$

(1944-1994)

AnalesdelIBUNAM

314

$65(64)$

(1930-1994)

ActaBotánicaMexicana

149

28(7)

(1988-1994)

CUADRO4. Temascubiertos porlastresrevistasconmayor regularidaden suaparición, durantelaúltimadécada

\begin{tabular}{lccc}
\hline Temas & $\begin{array}{l}\text { Boletín } \\
\text { SBM } \\
1984-1994\end{array}$ & $\begin{array}{l}\text { Anales } \\
\text { IBSerieBot. } \\
\text { 1984-1994 }\end{array}$ & $\begin{array}{l}\text { ActaBotánica } \\
\text { Mexicana } \\
1988-1994\end{array}$ \\
\hline Anatomía & 11 & 3 & 4 \\
Biologíafloral & 2 & 3 & \\
Biosistemática & 2 & 1 & 4 \\
Citogenética & 5 & 1 & 3 \\
Ecofisiología & 11 & 4 & \\
Ecología & 8 & 10 & \\
Etnobotánica & 5 & 6 & 1 \\
Fisiología & 1 & 1 & \\
Fitogeografía & 6 & 5 & 20 \\
Fitopatología & 3 & 13 & \\
Florística & 17 & 8 & 21 \\
Historia, biografía & & & \\
ybibliografía & 4 & 1 & 4 \\
Morfología & 4 & 1 & 4 \\
Nomenclatura & 4 & 5 & \\
Palinología & 5 & 5 & 4 \\
Taxonomía & 24 & 5 & \\
Vegetación & 5 & 5 & \\
Misceláneos & 5 & 5 & \\
\hline & & 5 & \\
\hline
\end{tabular}


En el Boletín se han publicado 396 artículos en 54 números a lo largo de sus primeros 50 años. Al revisar el contenido de éstos se aprecia, en primer término, la preferencia de los autores y la selección de los editores por temas taxonómicos, seguidos por florísticos, ecológicos, fitogeográficos y etnobotánicos.

Un análisis de la última década (a partir del número 44) indica que a través de numerosos artículos taxonómicos se han descrito un género y 34 especies de plantas nuevas para la ciencia, lo que ha contribuido al conocimiento de la diversidad de la flora de México.

En el cuadro 4, se pone de manifiesto que en las tres revistas comparadas hay ciertas tendencias generales en cuanto a los temas de mayor interés en estos últimos años. Si bien existe un mayor número de botánicos e instituciones participantes con trabajos publicados, se puede señalar que el mayor número de artículos en los temas prevalecientes, sigue recayendo sobre un grupo compacto de autores. A1gunos de los más productivos publican en las tres revistas indistintamente; otros, reflejan «lealtad» y afinidad por publicar sistemáticamente en una de ellas.

No hay que perder de vista que, coincidente con este mismo periodo de análisis (una década), se creó el Sistema Nacional de Investigadores de la Secretaría de Educación Pública, que promueve y orienta una parte de la producción científica en publicaciones de circulación internacional, editadas en el extranjero. Para el caso de los investigadores del Departamento de Botánica del Instituto de Biología, UNAM, este efecto se ilustra al encontrar que entre 1985 y 1990, en 57 revistas publicaron 212 artículos, de los cuales 90 se dieron a conocer en revistas nacionales (16 en el Boletín de la Sociedad Botánica de México) y 122 en revistas extranjeras (Lot, 1994).

En este sentido, hay que destacar que el Boletín mantiene su promedio de artículos y de autores por número anual, con todo y la aparición, hace 7 años, de Acta Botánica Mexicana, revista que por su mayor periodicidad ha logrado atraer y publicar un mayor número de contribuciones en los temas de taxonomía, florística y fitogeografía, en menos años que las otras revistas nacionales del área.

\section{CONSIDERACIONES FINALES}

El Boletín de la Sociedad Botánica de México, sin descuidar su finalidad de contribuir al conocimiento de la naturaleza del territorio nacional, en particular de la vegetación y flora del país, ha demostrado con la calidad de sus contribuciones, que realiza un esfuerzo serio por mejorar, en el sentido de lograr las metas de una revista competitiva internacionalmente. Sin embargo, no hay ninguna garantía de que dicha tendencia se mantenga o supere, ya que se trata de una revista subsidiada que depende de la vida azarosa de una sociedad científica. Es decir, que depende de la voluntad y capacidad de unas cuantas personas que en torno a la asociación hacen su mejor esfuerzo durante el periodo que toman la responsabilidad de conducir a la Sociedad y a su Boletín, como una parte de sus actividades profesionales y académicas.
En resumen, y reiterando lo señalado en el artículo «Destino de nuestras contribuciones botánicas: el caso de las revistas periódicas mexicanas» publicado en el Macpalxótil (Lot, 1994), el Boletín debería ser replanteado en el contexto de las revistas nacionales afines al área, de manera que pudiera definirse y complementarse mejor entre éstas, en bien de la comunidad científica y de la sociedad mexicana.

En lo particular, los editores del Boletín deberán insistir en que la regularidad de aparición se mantenga, y que sea superior a un número por año, para lograr que la publicación sea considerada periódica. Al mantener lo anterior, junto con un arbitraje serio y consistente, se satisfacen los requisitos de calidad, además de las expectativas de los autores y usuarios, lo que hace más profesional y atractiva la publicación.

Un concepto que valdría la pena considerar es el que propone la edición regular de números especiales, con artículos de revisión y síntesis sobre temas de gran actualidad que requieren ser discutidos ampliamente, sobre la base de reunir la mejor información disponible por especialistas invitados.

Finalmente, quisiéramos dejar un mensaje que no es exclusivo para el Boletín, sino para todas las revistas nacionales del área, y que se expresa en el siguiente párrafo, tomado de Lot (1994). Es verdaderamente necesario que los miembros de la comunidad científica tengan una imagen positiva de las publicaciones botánicas nacionales en el idioma castellano, pues en el fondo hay un acuerdo general en la búsqueda de la calidad. Una actitud negativa hacia nuestras revistas, generaría a la larga una baja en su calidad internacional, al obligar a los autores de los trabajos de mayor excelencia a ser enviados a otras revistas, donde sí serían justamente reconocidos y produciría un círculo vicioso que podría empobrecer y aún esterilizar la expresión nacional de nuestra ciencia. Una actitud positiva hacia dichas revistas, por el contrario, afirmaría el valor intelectual de nuestra cultura en el ámbito internacional.

\section{LITERATURA CITADA}

Benítez Bribiesca L, Bolívar A, Flores J. 1973. Memorias. Seminario sobre Revistas Científicas Nacionales. San Juan del Río, Qro. 28 a 30 de septiembre de 1972 . Centro de Servicios de Información y Documentación, CONACyT, México.

Bolívar JI, Brody T., Irwin CC, Fernández de la Garza G, Zamora P. 1972. Segunda Mesa Redonda. Los sistemas de información científica y técnica en México. En: Coloquio sobre Políticas Nacionales en Ciencia y Tecnología. Academia de la Investigación Científica, A.C., México.

Butanda A. 1989. XX Aniversario del Macpalxóchitl. Macpalxóchitl 122: $1-2$.

Larqué Saavedra A. 1993. La participación de las sociedades científicas para apoyar la investigación científica en las ciencias agrícolas en México. Ciencia (México) 44 (especial): 145-150.

Larqué Saavedra A. 1994. Revistas científicas en ciencias naturales. Bol. AIC. (México) 20: 12-14.

Lot A, Carvajal M. 1981. Cronología de los principales acontecimientos históricos de la Sociedad Botánica de México. Bol. Soc. Bot. México 40: 25-43 
Lot A 1994. Destino de nuestras contribuciones botánicas: el caso de las revistas periódicas mexicanas. Macpalxóchitl 133: 3-9.

Pérez Tamayo R, coord. 1988. Investigación e informacióncientíficas en México. Siglo Veintiuno Editores, México.
Sánchez y Gándara A, Magariños Lamas F, Wolf KB. 1992. El arte editorial en la literatura científica. UNAM, Ediciones Técnico Científicas, México. (Colección Biblioteca del editor) 